\title{
Analysis of Students' Understanding on Solar Eclipse Concept
}

\author{
Sahrul Saehana \\ Universitas Tadulako \\ Palu, Indonesia \\ sahrulsaehana@gmail.com \\ Darsikin \\ Universitas Tadulako \\ Palu, Indonesia
}

\author{
Unggul Wahyono \\ Universitas Tadulako \\ Palu, Indonesia
}

\begin{abstract}
The aim of this study was to analyze the understanding of students' concept on solar eclipse phenomena. The method of this study was a qualitative descriptive. This study was conducted for junior high school students at SMPN 21 Palu. The data were collected using multiple choice tests accompanied with Certain of Response Index (CRI) and interviews. The test was given before and after the solar eclipse occurred. This data was then calculated and analyzed to determine the percentage of understanding concept. Based on the analysis it can be concluded that students' understanding concept is in the medium category. A total solar eclipse phenomenon improves students' concepts. The study found misconceptions of the students in (1) position of the Sun, the Earth, and Moon during eclipse; (2) natural phenomena during solar eclipse; (3) phase of solar eclipse; and (4) safety methods on observing a solar eclipse.
\end{abstract}

Keywords-Student; CRI; solar eclipse concept.

\section{INTRODUCTION}

Solar eclipses happen when the moon moves between sun and earth, blocking the sun's rays and casting a shadow on earth. A total solar eclipse takes place when the moon completely covers the Sun and casts its umbra and penumbra on earth. A total eclipse of the sun can only take place when the moon is at perigee. On March $9^{\text {th, }}$ 2016, a total solar eclipse happened in Palu, Central Sulawesi (Indonesia).

During the total solar eclipse, some physical phenomena can be observed [1-4]. Reference [1] reported that this phenomenon affected to the meteorological parameters, such as solar radiation, temperature, and humidity. In comparing to a normal day, meteorological parameters changed during the solar eclipse, such as decreased in the UV and solar radiation, increased in relative humidity, and changed in air temperature and wind condition. Reference [5] also reported that gravitational acceleration was not significantly changed on Earth's surface [5].

Actually, the concept of a solar eclipse has been tough on junior high school. However, students still get difficulties in understanding of this concept. On the other hand, students' misconception was also found [6].
Ref. [6] reports that students' misconception can be found in daily life. Students considered solar eclipse occurred during the full moon phase. Students did not understand the position of the sun, moon, and earth when a solar eclipse occurred. In addition, students did not understand that solar eclipse occurred more frequently in a certain geographical area than the lunar eclipse.

The total solar eclipse occurred in Central Sulawesi, on March 9th, 2016, was a good chance for students to improve understanding of its concept. It can develop competence in order to explore and understand about scientific nature. So it can help learners to gain a deeper understanding of nature [7].

Observing a phenomenon directly (problem-based learning) can give significance experience to students [8-10]. This gives students to connect with the previous concept. Study on this and its relationship with other materials that are expected to improve the scientific literacy of students. Students' experiences can be obtained from direct observations of a solar eclipse through electronic and non-electronic media as well as social media.

This paper analyses students' understanding on solar total eclipse occurred on March 9th, 2016. Student pre-conception was identified and then it was analyzed. Students' understanding was tested before and after solar total eclipses phenomenon occurred.

\section{METHODS}

This study was a qualitative descriptive study.

This study was conducted before and after the phenomenon of a total solar eclipse on 5 March and 9 April 2016 at SMPN 21 Palu. Subjects in this study were students of class IX SMPN 21 , who have been studying the solar eclipse concept.

Students' conception was identified by using multiple choice instrument. Multiple choice tests accompanied by Certainly of Response Index (CRI) was used to determine the degree of understanding of the student concept. Then, by applying this test, respondent can be categorized in 
understanding (U), not understand (NU), and misconceptions (M) [15]. CRI scale and its criteria can be seen in Table 1.

TABLE 1. CRI AND ITS CRITERIA

\begin{tabular}{|c|l|c|c|}
\hline \multirow{2}{*}{ CRI } & \multicolumn{1}{|c|}{ Criteria } & \multicolumn{2}{|c|}{$\begin{array}{c}\text { Category of student } \\
\text { answered }\end{array}$} \\
\cline { 3 - 4 } & \multicolumn{1}{|c|}{$\begin{array}{c}\text { Correct } \\
0\end{array}$} & $\begin{array}{l}\text { Wrong } \\
\text { totally guessed answer: if respondent } \\
\text { problem by guessing }\end{array}$ & $\mathrm{MU}$ \\
\hline & $\begin{array}{l}\text { Almost guess: if respondent guessing } \\
\text { about 75\%-99\% }\end{array}$ & $\mathrm{NU}$ & $\mathrm{M}$ \\
\hline 2 & $\begin{array}{l}\text { Not sure: if respondent guessing about } \\
50 \%-74 \%\end{array}$ & $\mathrm{NU}$ & $\mathrm{M}$ \\
\hline 3 & $\begin{array}{l}\text { Sure: if respondent guessing about 25\%- } \\
49 \%\end{array}$ & $\mathrm{U}$ & $\mathrm{M}$ \\
\hline 4 & $\begin{array}{l}\text { Almost certain: if respondent guessing } \\
\text { about 1\%-24\% }\end{array}$ & $\mathrm{U}$ & $\mathrm{M}$ \\
\hline 5 & Certain: Not guessing & $\mathrm{U}$ & $\mathrm{M}$ \\
\hline
\end{tabular}

The test was applied before and after solar eclipse occurred. Analyzing data was done to determine the percentage of students in answering the question on the level of confidence in the percentage of the group understand, do not understand and misconceptions, by the following equation:

$$
\mathrm{Z}=\mathrm{f} / \mathrm{N} \times 100 \%
$$

where

$\mathrm{Z}=$ the percentage of student understanding

$\mathrm{f}=$ number of students per category

$\mathrm{N}=$ the sum of all student

Multiple choice instruments were used in Selected Respondent Test (TSR). In order to know students' understanding, Equation 1 was used to determine the degree of students' understanding. After, TSR applied, it was classified in the category of high, medium and low. Furthermore, interviewed respondents were selected based on the degree of student understanding in Table 2.

TABLE 2 QUALIFYING TEST RESULTS

\begin{tabular}{|c|c|}
\hline Score Interval $(\%)$ & Category \\
\hline $66,68 \leq \mathrm{Z} \leq 100$ & High \\
\hline $33,34 \leq \mathrm{Z} \leq 66,67$ & Medium \\
\hline $0 \leq \mathrm{Z} \leq 33,33$ & Low \\
\hline
\end{tabular}

Deep interview with respondents was also done in order to check students' consistency. The interview was done for all categories responded. Transcript of interviewing was used for analyzing results of students' understanding.

\section{RESULTS AND DISCUSSION}

The instrument used in this paper consisted of 10 questions and they have been validated by experts. The test was accompanied with certainty response index (CRI). Then, it was given to students of class IX at SMPN 21 Palu before the solar eclipse. After the total solar eclipse occurred, the students' conception was tested again. Calculating results of students' understanding of the concept of solar eclipse phenomena were presented in Table 3. The grouping respondent who become participants (or interviewing respondent) is presented in Table 4.

TABLE 3 ANALYSING OF STUdENT UNDERSTANDING OF SOLAR ECLIPSE CONCEPT

\begin{tabular}{|c|c|c|c|c|c|c|}
\hline \multirow{2}{*}{ Number } & \multicolumn{3}{|c|}{ Before GMT } & \multicolumn{3}{c|}{ After GMT } \\
\cline { 2 - 7 } & $\mathrm{U}(\%)$ & $\begin{array}{c}\text { NU } \\
(\%)\end{array}$ & $\mathrm{M}(\%)$ & $\mathrm{U}(\%)$ & $\begin{array}{c}\text { NU } \\
(\%)\end{array}$ & $\mathrm{M}(\%)$ \\
\hline 1 & 46.67 & 33.33 & 20.00 & 53.33 & 13.33 & 33.33 \\
\hline 2 & 13.33 & 66.67 & 20.00 & 20.00 & 20.00 & 60.00 \\
\hline 3 & 66.67 & 26.67 & 6.67 & 80.00 & 6.67 & 13.33 \\
\hline 4 & 26.67 & 40.00 & 33.33 & 53.33 & 33.33 & 13.33 \\
\hline 5 & 46.67 & 33.33 & 20.00 & 86.67 & 6.67 & 6.67 \\
\hline 6 & 33.33 & 26.67 & 40.00 & 40.00 & 13.33 & 46.67 \\
\hline 7 & 20.00 & 73.33 & 6.67 & 20.00 & 46.67 & 33.33 \\
\hline 8 & 13.33 & 60.00 & 26.67 & 13.33 & 46.67 & 40.00 \\
\hline 9 & 0.00 & 60.00 & 40.00 & 33.33 & 13.33 & 53.33 \\
\hline 10 & 0.00 & 86.67 & 13.33 & 0.00 & 46.67 & 53.33 \\
\hline & $\mathbf{2 6 . 6 7}$ & $\mathbf{5 0 . 9 1}$ & $\mathbf{2 2 . 4 2}$ & $\mathbf{3 8 . 7 9}$ & $\mathbf{2 5 . 4 5}$ & $\mathbf{3 5 . 7 6}$ \\
\hline
\end{tabular}

TABLE 4 GROUPING OF RESPONDENTS AS A PARTICIPANT

\begin{tabular}{|c|c|c|c|c|}
\hline \multirow{2}{*}{ Respondent } & \multicolumn{3}{|c|}{ After GMT } & \multirow{2}{*}{ Category } \\
\cline { 2 - 4 } & $\mathrm{U}(\%)$ & $\mathrm{NU}(\%)$ & $\mathrm{M}(\%)$ & \\
\hline R-25 & 72.73 & 9.09 & 18.18 & High \\
\hline R-33 & 54.55 & 27.27 & 18.18 & Medium \\
\hline R-21 & 27.27 & 9.09 & 63.64 & Low \\
\hline
\end{tabular}

Problem 1: Understanding the position of the sun, earth, and moon during a solar eclipse.

Before the solar eclipse, about $33.33 \%$ of the students did not know the position of the sun, earth, and the moon during a solar eclipse. However, about $20 \%$ of the student had misconceptions. Misconception respondents (R21) assumed that a solar eclipse occurs when the sun covered the entire month. It can be known from interviewed below.

\section{Researcher: Why did you say A (position of the sun was between earth and the moon) that resulted in a total solar eclipse? \\ Respondents: Because of my knowledge, the sun was over the moon to the earth looks dark \\ Researcher: So, in your opinion, when did total solar eclipse occurs? \\ Respondents: When the moon covered the entire section, e sorry, when the sun cover all parts of the moon}

Based on Table 3, understanding about the position of sun, moon, and earth was increased from $46.67 \%$ to $53.33 \%$. On the other hand, misconception was also increased from $20.00 \%$ to $33.33 \%$. 
Problem 2: Understanding the natural phenomenon during a solar eclipse.

Before the solar total eclipse, about $66.67 \%$ of students do not understand about natural phenomenon during a solar total eclipse. They assumed that during a solar eclipse, the temperature was not decreased. They also assumed that unsafe to see a solar eclipse directly.

After the solar total eclipse, the percentage of student understanding did not increase significantly (from $13.33 \%$ to $20.00 \%$ ). Interestingly, after solar total eclipse occurs, misconception student was increased (from 20\% to 60\%).

By interviewing respondent (R-33), it was known misunderstanding about the temperature during a solar eclipse were done. The respondent assumed that temperature was not the decreases. Their opinion also related to the mythology of solar eclipse [16-19]. The opinion of respondent was also wrong about safety to observe the solar total eclipse. Most of them thought that it was saved observing the total solar eclipse directly.

In the opinion of the respondent (R-21) that animal behaviors did not change when the total solar eclipse occurred. It may be observed was not done by the respondent when the solar total eclipse occurred.

The misconception also happened in period and frequency of solar total eclipse. They thought that solar total eclipse was more often than a lunar eclipse. In fact, the lunar eclipse will happen about two till five times a year.

Problem number 3: Understanding the phases of a solar eclipse.

About $26.67 \%$ of student cannot understand phase of the solar eclipse. They got difficulties in describing the phase of the solar eclipse. Only $6.67 \%$ of the student was a misconception. After solar eclipse occurred, the number of both reduced.

Problem Number 4: Analyzing the images solar eclipse observation position on Earth.

Observing the solar total eclipse was effective in studying the image of it. The number of students who were not understanding decreased from $33.33 \%$ to $6.67 \%$. On the other hand, misconception student also reduced from $20.00 \%$ to $6.67 \%$.

Misconceptions student assumed that the solar eclipse observation position in the rear position of the earth (night) because it is described in the hemisphere darkened, hinted solar eclipse darkened.

Based on students interview, a student in lower category assume that position behind the earth appears dark (night) is where the solar eclipse observation. Here are excerpts of interviews with R-21:

Researcher: If in section $B$ (position hemisphere night) whether there is something or what can we observe?
Respondents:

Researcher: Respondents:

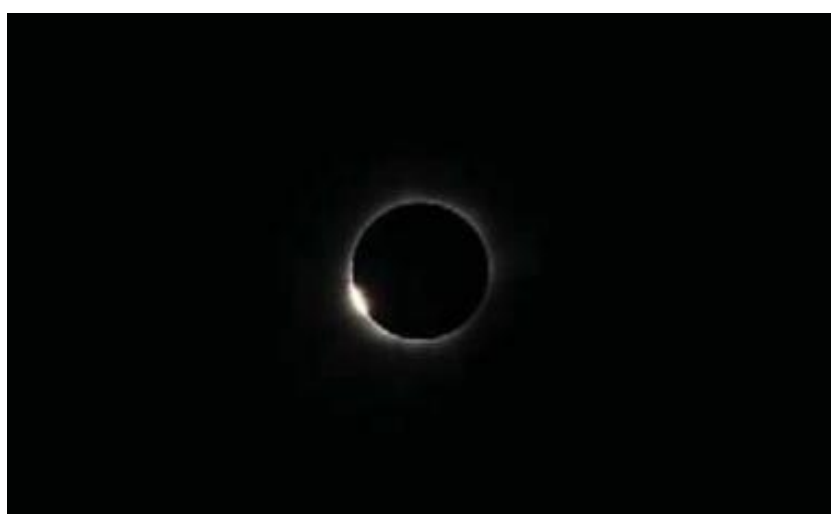

Fig. 1. Solar total eclipse [12]

Problem number 5: Understanding phase partial solar eclipse.

Before the solar eclipse, about $33.33 \%$ of students do not understand. Each student assumes that the phase of the picture is the phase of a lunar eclipse, the total solar eclipse of the sun and the ring. Student misconceptions were about $20.00 \%$ thought that the solar eclipse ring. Students thought that moon covered the sun (in part) was an eclipse of the moon.

After the solar eclipse, the percentage of the not understood student was reduced to $6.67 \%$. Interviewing result showed that participants understand the disc in only cover part of the solar disc that includes partial solar eclipse.

Problem number 6: understanding phase indicator ring of a solar eclipse.

Before the eclipse of the sun, the percentage of a student about $26.67 \%$ who has chosen an incorrect option and categorized as a misconception. Nearly, $40 \%$ of the students did not understand of a solar eclipse.

After the solar total eclipse, misconception student was reduced to $13.33 \%$. Interviews showed that $46.67 \%$ participants think that image was a phase of the ring of a solar eclipse which is smaller than the disc in the solar disc cover that looks like a ring.

Only the student participants and student categories are being considered in the right lower category covered the sun, causing a total solar eclipse. Here are excerpts of interviews with R-33:

Researchers: Problem number seven you saw

Respondents: $\quad$ The total solar

Researchers: Why do you say the sun is a total eclipse? 
Respondents:

Because the total fitting, so the sun was covered, a total solar eclipse occurred, yes closed all.

Problem number 7: Analyzing diagram drawing ring solar eclipse, partial and total eclipses.

Before eclipse, about $73.33 \%$ of students did not understand about ring solar eclipse, partial and total eclipses. Their incorrect answer was chosen with low certainty.

After the solar eclipse, misunderstanding student was increased to $33.33 \%$. They assumed total eclipse was the solar eclipse rings and it was because the position of the moon does not cover the full sun. They not convinced and have not mastered the material diagram of the solar eclipse.

According to interviews with participants gave correct answers assume that the total solar eclipse was dark, only the edge of the ring solar eclipse the moon appears bright disc and solar eclipses dark as only partially. However, participants do not see the position of the moon between the earth and the sun have different distances. The solar eclipse of the moon positions closer to the earth and the solar eclipse ring far enough away from the Earth.

Problem number 8: safety observing a solar eclipse.

Before the eclipse of the sun, about $60.00 \%$ of students did not understand the safe way of observing a total solar eclipse.They also didn't know the negative effect on observing eclipse directly. They think that observed on the surface of the water is safe manner. Likewise, about $26.67 \%$ of students were misconceptions.

After the solar eclipse was occurred, not understand student was decreased. However, misconception of the student was increased. They thought that the solar eclipse observed on the surface of the water is a safe way to observe a solar eclipse because the sun was observed at the water surface just a reflection not directly so that water is an intermediary to observe a solar eclipse.

Observing the solar eclipse on the water surface reflects ultraviolet B rays at $3 \%-13 \%$ so that it can burn the retina of the eye and cause blindness [11-12]. So, All of the participants thought that the projection pin-hole an unsafe manner, because radiation from the sun during an eclipse can affect the eye pass through a small hole. Here are excerpts of interviews with students of the high category:

\section{Researcher: Why do you say there are safe?}

Respondents: Pinhole, circular pin-hole although small but we were affected by radiation from the sun, so it is not safe

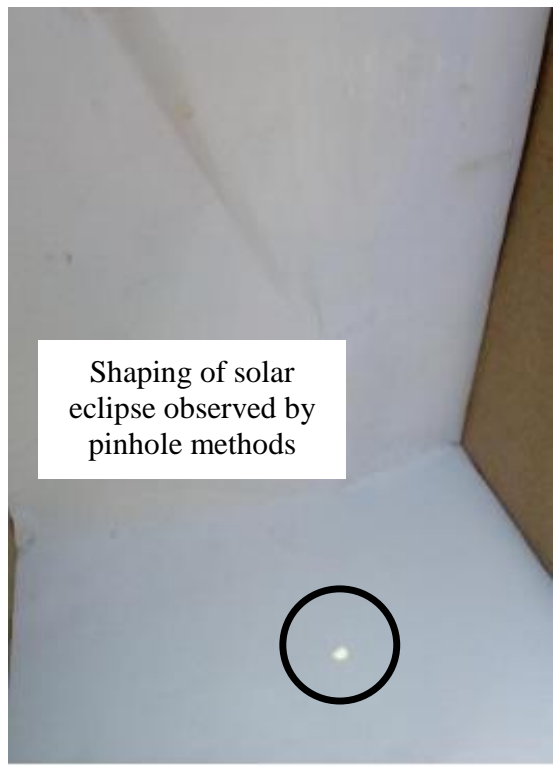

Fig. 2 Observation of solar eclipse by pinhole methods.

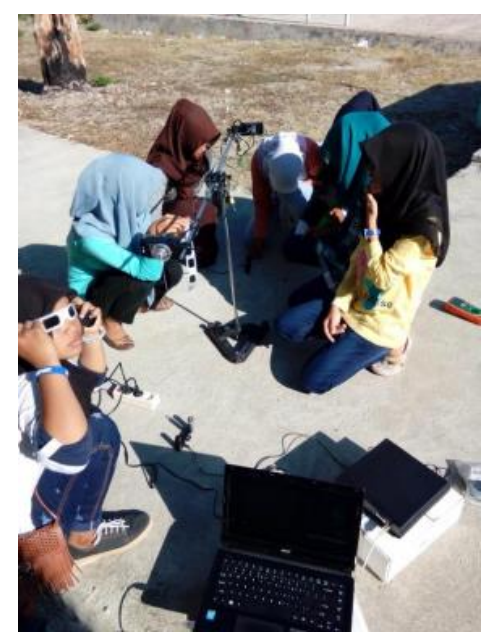

Fig. 3 Observation of solar eclipse by using sunglasses.

Problem number 9: Understanding the Moon shadow direction when eclipse appeared.

Before the eclipse, about $40 \%$ of students were misconceptions. They think the direction of the moon's shadow during an eclipse occurred following the direction of sunrise and sunset (east to west). Then, after the solar eclipse, understanding of student was increased to $33.33 \%$

The participants think shadows month direction from west to east. Following the sun directions were from the west. Here are excerpts of interviews with R-21:

$\begin{array}{ll}\text { Researcher: } & \text { At the time of the solar eclipse shadow } \\ & \text { first appeared from what direction? } \\ \text { Respondents: } & \text { In accordance with the direction of the } \\ & \text { rising sun from west to east } \\ \text { Researcher: } & \text { Why do you say from west to east? }\end{array}$


Respondents: $\quad$ There's always the sun from east to west, so that the direction the sun rises right out of the west

Problem number 10: Understanding the current moon phase indicator of a solar eclipse.

Before the eclipse of the sun, as much as $86.67 \%$ of students do not understand the pure conjecture assumes when the first quarter moon phase a solar eclipse. On the other hand, about $13.33 \%$, the student was a misconception. A student can not understand this concept at all.

After the solar eclipse, not understand students was about $46.67 \%$. However, student misconceptions were increased. They think a solar eclipse can be observed when the phase of the moon in the first quarter. Similarly, previous studies show that misconceptions student think solar eclipses occur when the moon is not the new moon phase. It can be concluded that students level of confidence about moon phase during a solar eclipse was increased by observing solar total eclipse [13].

According to interviews with participants (R-25 and R-21), they considered phases of the moon during an eclipse occurred in the first quarter phase. It can be caused a solar eclipse occurs for the first time in Palu so they have no experience before. Participants R-33 assumed when the full moon appears larger than the other phases of the moon. Participant does not understand the current phase of the moon and the new moon occurs assume 360 years later. It was also assumed when the moon for the lunar eclipse and new moon when the position of the sun, the moon, and Earth line up on the field ecliptic.

Trundle, Atwood, and Christopher (2002) reported that a great majority of student-teachers had misconceptions about the phases of the moon. In their comparative study with participants from China and New Zealand, Bryce and Blown (2012) found that primary school student-teachers and primary school teachers in both countries lacked the academic jargon to explain - and the skills to model - astronomy subjects, and they had inadequate training in astronomy in general.

\section{CONCLUSION}

Students' understanding total solar eclipses concept is in the medium category. Total solar eclipse phenomenon improves student's concepts. It was also found misconception of the student: (1) position of the Sun, the Earth, and the Moon during eclipse; (2) natural phenomenon during solar eclipse; (3) phase of solar eclipse; and (4) safety methods on observing a solar eclipse.

\section{ACKNOWLEDGMENT}

The author would like to thanks to Dean FKIP UNTAD for supporting funding and facility of this research. Appreciating also gives to Harianto Andi Matu as the assistantship for this research.

\section{REFERENCES}

[1] B. Paramitha et al., "Changes in Meteorological Parameters (i.e. UV and Solar Radiation, Air Temperature, Humidity and Wind Condition) during the Partial Solar Eclipse of 9 March 2016," IOP Conf. Series: Mat. Sci. Eng. Vol. 180 p. 012131, 2017.

[2] L. S. Riza et al., "Analysis of atmospheric pressure, temperature, and wind speed profiles during total solar eclipse 9 March 2016 using time series clustering," J. Phys. Conf. Ser., vol. 771, p. 12009, 2016.

[3] A. Laesanpura et al., "Micro-gravity measurements during the total solar eclipse of 9 March 2016 in Indonesia,” J. Phys. Conf. Ser., vol. 771, p. 12003, 2016

[4] S. S. Kumar and R. Rengaiyan, "Influence of solar eclipse on seawater," Nat. Sci., vol. 3, no. 1, pp. 69-74, 2011.

[5] M. G. Nugraha et al., "Influence of Partial Solar Eclipse 2016 on the surface gravity acceleration using photogate sensor on Kater's reversible pendulum,” J. Phys. Conf. Ser., vol. 771, p. 12002, 2016.

[6] K. Kiroglu, "Students Are Not Highly Familiar with Astronomy Concepts-But What about the Teachers?,” J. Educ. Train. Stud., vol. 3, no. 4, pp. 31-41, 2015.

[7] E. Whitelegg and M. Parry, "Real-life contexts for learning physics: meanings, issues, and practice," Phys. Educ., vol. 34, no. October, pp. 68-72, 1999.

[8] A. T. Handini, Y. Yulianty, P. W. Premadi, and A. Annafi, "UNAWE Indonesia project: raising total solar eclipse 2016 awareness through educational packages,” J. Phys. Conf. Ser., vol. 771, p. 12022, 2016.

[9] S. Fatima et al., "Total solar eclipse education for young generation at Palangkaraya, Central Kalimantan,” J. Phys. Conf. Ser., vol. 771, no. June 2013, p. 12044, 2016.

[10] A. Yamani et al., "Chasing the shadows, a trip to spice island," J. Phys. Conf. Ser., vol. 771, p. 12042, 2016.

[11] E. P. Raharja and Y. Pramudya, "Outreach activities in anticipation of the 2016 solar eclipse in Sorong,” J. Phys. Conf. Ser., vol. 771, p. 12024, 2016.

[12] A. P. Rachmadian, C. Kunjaya, W. Wahono, and A. A. Anugrah, "Public outreach and education during the 2016 total solar eclipse in Palu and Malang," J. Phys. Conf. Ser., vol. 771, p. 12023, 2016.

[13] J. M. Pasachoff, "Scientific observations at total solar eclipses," Res. Astron. Astrophys., vol. 9, no. 6, pp. 613-634, 2009.

[14] S. E. Lenhard, "Utilizing Problem-Based Learning Activities in Science with Students with Disabilities," 2015.

[15] S. Hasan, D. Bagayoko, and E. L. Kelley, "Misconceptions and the Certainty of Response Index (CRI),” Phys. Educ., vol. 34, pp. 294-299, 1999.

[16] S. S. Kumar and R. Rengaiyan, "Vedic mythology of solar eclipse and its scientific validation," vol. 13, no. October, pp. 716-724, 2014.

[17] S. N. Ball, a Mishra, a K. Dutta, and P. Sen, "International Conference on Recent Trends in Physics (ICRTP 2012)," J. Phys. Conf. Ser., vol. 365, p. 11001, 2012.

[18] I. Baig, S. Ahmed, M. Zeeshan, C. Author, and I. Baig, "Myths and superstitions related to eclipses," vol. 132, no. 10, pp. 250-254, 2007. 